\title{
Acute Effects of Liver Vein Occlusion by Stent-Graft Placed in Transjugular Intrahepatic Portosystemic Shunt Channel: An Experimental Study
}

\author{
Inger Keussen, ${ }^{1}$ Lennart Bergqvist, ${ }^{2}$ Pehr Rissler, ${ }^{3}$ Wojciech Cwikiel ${ }^{4}$ \\ ${ }^{1}$ Department of Radiology, University Hospital, Lund, Sweden \\ ${ }^{2}$ Department of Radiation Physics, University Hospital, Lund, Sweden \\ ${ }^{3}$ Department of Pathology, University Hospital, Lund, Sweden \\ ${ }^{4}$ Department of Radiology, University of Michigan, Ann Arbor, Michigan, USA
}

\begin{abstract}
The purpose of this study was to evaluate the effects of hepatic vein occlusion by stent-graft used in transjugular intrahepatic portosystemic shunt (TIPS). The experiments were performed in six healthy pigs under general anesthesia. Following percutaneous transhepatic implantation of a porta-cath in the right hepatic vein, TIPS was created with a stent-graft (Viatorr; W L Gore, Flagstaff, AZ, USA). The outflow from the hepatic vein, blocked by the stent-graft was documented by injection of contrast medium and repeated injections of ${ }^{99} \mathrm{Tc}^{\mathrm{m}}$-labeled human serum albumin through the port-a-cath. After 2 weeks, the outflow was reevaluated, the pigs were sacrificed, and histopathologic examination of the liver was performed. Occlusion of the hepatic vein by a stent-graft had a short and temporary effect on the outflow. Histopathological examination from the affected liver segment showed no divergent pattern. Stentgrafts used in TIPS block the outflow from the liver vein, but do not have a prolonged circulatory effect and do not affect the liver parenchyma.
\end{abstract}

Key words: Portal hypertension-Transjugular intrahepatic portosystemic shunt-Stent-graft-Hepatic vein occlusion

Over the last 10 years, transjugular intrahepatic portosystematic shunt (TIPS) procedures have almost completely replaced surgical portosystemic shunts in treatment of patients with portal hypertension and repeated bleeding from gastroesophageal varices [1-5] or refractory ascites. Long-

Correspondence to: Inger Keussen; email: inger.keussen@skane.se term results of TIPS are, however, unsatisfactory due to the development of pseudointimal hyperplasia in the shunt channel and/or intimal hyperplasia in the adjacent hepatic vein [6].

To overcome these problems, stent-grafts have been used [7-12]. These stent-grafts separate the shunt channel from the liver tissue, bile ducts, and the wall of the hepatic vein, preventing development of hyperplasia.

Following TIPS, blood from the portal vein passes through the created shunt toward the right atrium, whereas flow to the intrahepatic portal vein branches is substantially decreased [13]. The cranial portion used for the TIPS stentgraft might block the outflow from the hepatic vein to which the shunt extends. A decrease in the hepatic arterial flow to the liver segment drained by the blocked liver vein was reported [14] as well as segmental liver ischemia [15]. Patients treated with a TIPS often have an impaired liver function. Reduced blood flow through one liver segment can cause further deterioration.

The present study was performed in order to evaluate if the outflow from the hepatic vein blocked by a stent-graft, causing a "pseudo-Budd-Chiari-syndrome," changed over 2 weeks of time and if there were any possible consequences for the affected liver segment.

\section{Materials and Methods}

\section{Animals and Anesthesia}

Six healthy pigs, 36-40 kg body weight, were used for the experiments after approval from the local ethics committee for animal research. All procedures were performed under general anesthesia, induced by intramuscular injection of $2 \mathrm{mg} / \mathrm{kg}$ azap- 
erone (Stresnil Vet; Leo, Helsingborg, Sweden), followed by intravenous injection of 2-4 mL thiopental (Pentothal Natrium; Abbott Lab, N. Chicago, IL, USA) 5\% solution. Following intubation, anesthesia was maintained by artificial ventilation with a mixture of oxygen and nitric oxide and continuous infusion of ketamin-hydrocloride (Ketalar; Parke-Davis, Solna, Sweden), $1 \mathrm{mg} / \mathrm{min}$, and midazolam (Dormicum; Roche, Stockholm, Sweden), $5 \mathrm{mg} / \mathrm{h}$. At the end of the experiments, all animals were sacrificed by an intracardial injection of an overdose of potassium.

\section{Preparation of Radiopharmaceuticals}

Human serum albumin (HSA) labeled to ${ }^{99} \mathrm{Tc}^{\mathrm{m}}$ is frequently used for circulation and blood flow studies. In our study, we used TechneScan ${ }^{\circledR}$ HSA (Mallinckrodt Medical B.V., Petten, Holland), which was labeled to ${ }^{99} \mathrm{Tc}^{\mathrm{m}}$ according to the manufacturer's instructions. Freeze-dried HSA was dissolved in $8-10 \mathrm{~mL}$ pertechnetate solution (406-1790 MBq). After a 15-min incubation time, the preparation was ready to use. All preparations were tested within $5 \mathrm{~h}$ after labeling with instant thin-layer chromatography (ITLC-SG; Gelman Sciences, Ann Arbor, MI, USA), using $1 \times 8$-cm strips and 2-butanone (ethyl-methyl-ketone) as the mobile phase. The amount of free pertechnetate varied between $0.04 \%$ and $0.45 \%$ (mean: $0.25 \%$ ). The injected activity was determined by measurements of the syringe before and after injection in a radioisotope calibrator (CRC-35R; Capintec, Pittsburgh, PA, USA). Blood samples were weighed and measured for radioactivity in a gamma counter (1282 CompuGamma CS; Wallac Oy, Turku, Finland). Assuming that the blood volume in pigs was $8 \%$ of the body weight, the percentage of the injected radioactivity leakage to peripheral blood was calculated.

\section{Procedures}

The left jugular vein was punctured after surgical cut down and thereafter catheterised. The tip of a $5 \mathrm{~F}$ Cobra catheter (Cordis, Johnson \& Johnson CO, Roden, Netherlands) was manipulated jointly with a 0.035 glidewire (Terumo, Tokyo, Japan) into a peripheral branch of the right hepatic vein. Subsequently the tip of the catheter was used as a target for the percutaneous transhepatic puncture of this vein. The puncture was performed using Seldinger technique, with a 20G needle (Mediplast, Malmoe, Sweden), subsequently replaced over a 0.018 -in. Cope wire by a $5 \mathrm{~F}$ catheter (transhepatic cholangiography catheter cannula, both from William Cook Europe, Bjaeverskov, Denmark). Contrast injection (Omnipaque, $350 \mathrm{~mL}$ I/mL; Amersham Health, Oslo, Norway) confirmed free outflow from the right hepatic vein.

Thereafter, a subcutaneous reservoir (port-a-cath; Deltec, Smiths Medical Industries, St. Paul, MN, USA) was implanted subcutaneously at the level of the liver, with the tip of the catheter placed in the right hepatic vein, approximately $5 \mathrm{~mm}$ from the confluence into the inferior vena cava. A transjugular, transhepatic access to the portal vein was created with a Cook TIPS Set (William Cook Europe). Through the port-a-cath, the radionuclide ${ }^{99} \mathrm{Tc}^{\mathrm{m}}$-HSA was injected into the right hepatic vein. Samples of venous blood from the peripheral circulation was obtained after 1, 3, 5 and $10 \mathrm{~min}$, before the TIPS was created.

The TIPS was created with a $10 \times 40-\mathrm{mm}$ stent-graft, (VIATORR; W L Gore, Flagstaff, AZ, USA). The stent-graft was dilated with a $10 \times 40-\mathrm{mm}$ balloon (MediTech; Boston Scientific,
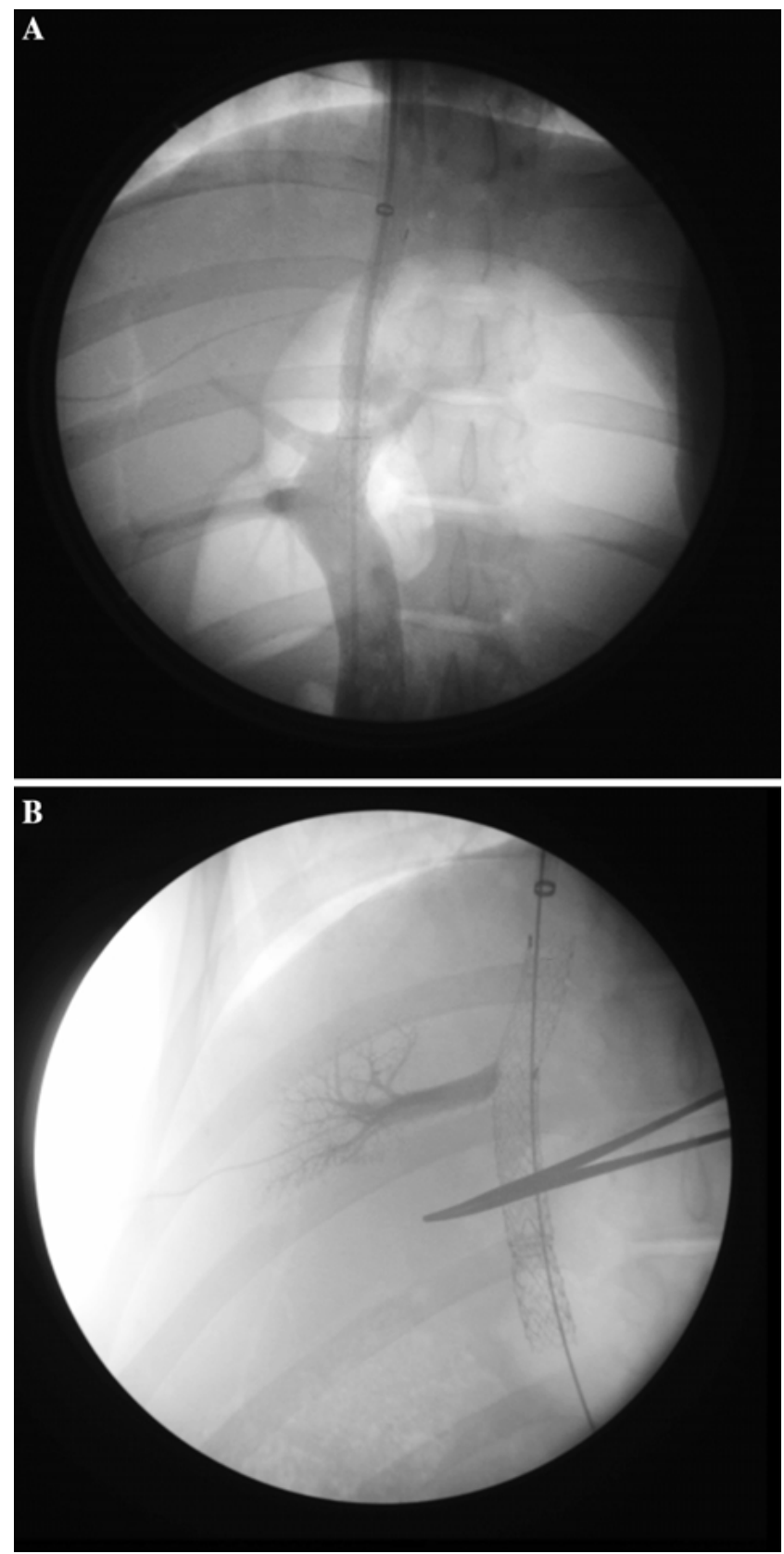

Fig. 1. (A) Portography after TIPS shows blood flow through the TIPS channel. (B) Percutaneous contrast injection show blockage of the right hepatic vein after TIPS.

Watertown, MA, USA). Free flow through the TIPS channel was confirmed by portography (Fig. 1A). Occlusion of the right hepatic vein by the inserted stent-graft was documented by injection of contrast medium in the port-a-cath (Fig. 1B). Injection of ${ }^{99} \mathrm{Tc}^{\mathrm{m}}$ HSA through the port-a-cath in the occluded right hepatic vein was followed by blood sampling from the peripheral vein after 1, 3, 5, and 10 minutes.

A repeated injection of ${ }^{99} \mathrm{Tc}^{\mathrm{m}}$-HSA as described earlier was planned for 2 weeks later. Unfortunately, all of the port-a-caths had dislodged from the intended location in the right hepatic veins. Under fluoroscopic guidance, percutaneous puncture of the right 


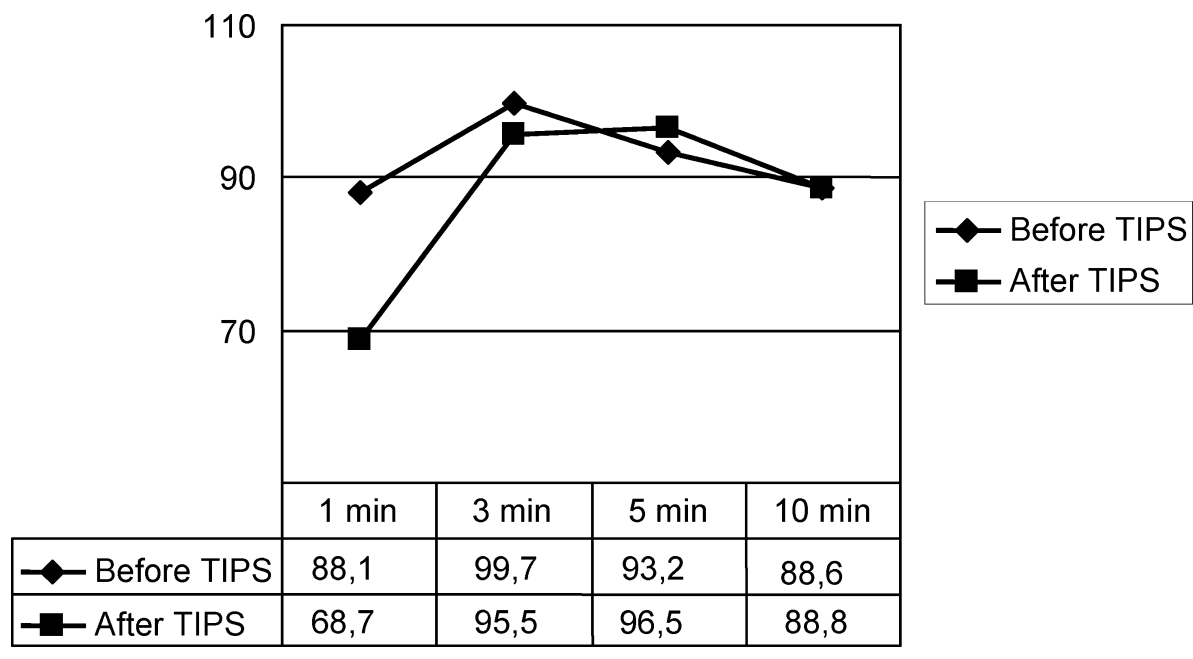

Fig. 2. Measurements of ${ }^{99} \mathrm{Tc}^{\mathrm{m}}-\mathrm{HSA}$ in peripheral blood before and after occlusion of the hepatic vein with a stent-graft; mean values. hepatic vein was performed with a $20 \mathrm{G}$ needle, toward the cephalic portion of the stent-graft. The position of the needle in the occluded hepatic vein was confirmed by contrast injection. The ${ }^{99} \mathrm{Tc}^{\mathrm{m}}$-HSA was thereafter injected through the needle. The sampling of peripheral blood was repeated in the same intervals as previously.

Following sacrifice of the animals, parts of the liver, including segments with the occluded hepatic vein, stent-graft, and normal liver from the left lobe were resected and sent for histopathological examination for evaluation of possible changes secondary to venous congestion. Three samples from each liver lobe were evaluated. The liver tissue was fixed in $10 \%$ natural buffer formalin and embedded in paraffin. Slides were prepared and stained with hematoxylin and eosin according to standard protocols.

\section{Results}

Despite successful insertion of the stent-grafts in the intended position, the hepatic vein was not completely blocked in one pig due to an anatomic variation and this pig was excluded from the study. In one pig, the placed stentgraft was too short and its bare part did not reach far enough into the portal vein. This portion of the shunt was therefore supported with a $12 \times 80-\mathrm{mm}$ stent (Smart, Cordis) to obtain optimal blood flow through the shunt channel. One pig died of respiratory failure just prior to the final radionuclide examination. Consequently, the experiments could only be completed in four pigs, which excluded the possibility of statistical evaluation at the end point of the study.

The radionuclide examination after TIPS showed primarily (after $1 \mathrm{~min}$ ) decreased outflow from the occluded hepatic vein, compared to the measurements before TIPS. However, after $3 \mathrm{~min}$, the outflow was normalized (Fig. 2). Values obtained in four pigs after 2 weeks were comparable to the ones obtained before TIPS, indicating good outflow from the liver vein closed by the stent-grafts. There were no complications secondary to dislodgement of the subcutaneous reservoirs from the liver.

The stent-grafts were fully patent in one pig; the remaining were occluded to $75-100 \%$.
One animal showed macroscopic cirrhosis, whereas the others showed no macroscopic fibrosis, cirrhosis, steatosis, or sign of congestion. Most of the samples had a microscopically similar pattern with normal acinar structure. There were no marked portal fibrosis, no bile-duct damage, and no interface hepatitis. A few neutrofils, lymphocytes, and plasma cells were found in the portal tract. Terminal hepatic venule and sinusoids showed minor dilatation but no fibrosis around central veins.

One pig had a different histopathological finding. This pig had signs of cirrhosis with marked infiltration of neutrophils, lymphocytes, and plasma cells in both samples, findings most likely related to chronic defects. In two more samples, from different pigs and from different lobes, light portal fibrosis was found with a mild infiltration of neutrophils, lymphocytes, and plasma cells, but no fibrosis around central veins.

\section{Discussion}

The advantage of stent-grafts in TIPS is its ability to separate the shunt channel from the liver parenchyma, bile ducts, and the wall of the hepatic vein, preventing development of pseudointimal and intimal hyperplasia $[2,4,11]$. The stentgraft has also been used to treat hemorrhage after extrahepatic portal vein puncture during a TIPS procedure [16]. Several graft materials have been used for construction of stent-grafts [8-10]. The Viatorr stent-graft (W L Gore, Flagstaff, AZ, USA) used in our study is a nitinol-polytetrafluoroethylene stent-graft for TIPS, commercially available and easy to handle [17]. These dedicated stent-grafts can also be safely used in patients who need a TIPS while waiting for liver transplantation [18].

Changes in the hepatic perfusion of the liver occur after TIPS placement [13]. The blood flow to the intrahepatic portal vein branches is diminished and the liver is supplied with blood mainly from the hepatic artery. The cranial portion of a stent-graft used for TIPS, positioned in a hepatic vein, blocks the outflow from this vein $[14,15]$. It has been 
shown that the use of stent-grafts can decrease perfusion to the adjacent liver segment, due to occlusion of a liver vein [14]. This can potentially have serious consequences for a patient with an already impaired liver function. In cirrhotic patients, computed tomography has revealed segmental liver ischemia in a few cases [15] remaining for a long time.

In our experiment, we wanted to evaluate venous outflow from the liver segment, drained by hepatic vein, which became occluded by the stent-graft used for TIPS. Activity of ${ }^{99} \mathrm{Tc}^{\mathrm{m}}$-HSA injected in the occluded liver vein measured in peripheral blood was primarily slightly decreased. However, in our experiments, the activity reached the same level after $3 \mathrm{~min}$ as that measured before TIPS. Our findings indicated that occlusion of one liver vein affected the venous outflow only for a very short period of time when the other liver veins remained open. The measurements obtained after 2 weeks confirmed these findings.

Histopathological assessment showed normal acinar structure in most of the samples. One pig had a divergent pattern, probably due to more chronic changes. Two pigs showed a very light increase of fibrosis, which might be within normal anatomical variation.

Statistical evaluation of our material was not possible because completion could only be performed in four animals due to the complications described earlier. Another limitation of the study is, of course, that it was performed in healthy animals and the findings can be different in patients with cirrhosis.

We conclude in our limited experimental study that a "pseudo-Budd-Chiari syndrome," which appeared after insertion of a stent-graft in the TIPS channel, had no significant impact on venous outflow. Occlusion does not cause obvious changes in the affected liver segment.

\section{References}

1. Richter GM, Noeldge G, Palmaz JC, et al. (1990) Transjugular intrahepatic portocaval stent shunt. Preliminary clinical results. Radiology 174:1027-1030

2. Haskal ZJ, Ring EJ, LaBerge JM, et al. (1992) Role of parallel transjugular intrahepatic portosystemic shunts in patients with persistent portal hypertension. Radiology 185:813-817
3. Burroughs AK, Patch D (1999) Transjugular intrahepatic portosystemic shunt. Semin Liver Dis 19:457-473

4. Rees CJ, Nylander DL, Thompson NP, et al. (2000) Do gastric and oesophageal varices bleed at different portal pressures and is TIPS an effective treatment? Liver 20:253-256

5. Lake JR (2000) The role of transjugular portosystemic shunting in patients with ascites. N Engl J Med 342:1745-1747

6. Saxon RR, Ross PL, Mendel-Hartvig J, et al. (1998) Transjugular intrahepatic portosystemic shunt patency and the importance of stenosis location in the development of recurrent symptoms. Radiology 207:683-693

7. Haskal ZJ, Brenneke LJ (1999) Porous and nonporous polycarbonate urethane stent-grafts for TIPS formation: biologic responses. J Vasc Intervent Radiol 10:1255-1263

8. Haskal ZJ, Davis A, McAllister A, et al. (1997) PTFE-encapsulated endovascular stent-graft for transjugular intrahepatic portosystemic shunts: experimental evaluation. Radiology 205:682-688

9. Cejna M, Thurnher S, Pidlich J, et al. (1999) Primary implantation of polyester-covered stent-grafts for transjugular intrahepatic portosystemic stent shunts (TIPSS): a pilot study. Cardiovasc Intervent Radiol 22:305-310

10. Tanihata H, Saxon RR, Kubota Y, et al. (1997) Transjugular intrahepatic shunt with silicone-covered Wallstents: results in a swine model. Radiology 205:181-184

11. Andrews RT, Saxon RR, Bloch RD, et al. (1999) Stent-Grafts for de novo TIPS: technique and early results. J Vasc Intervent Radiol 10:1371-1378

12. Cejna M, Peck-Radosavljevic M, Thurnher S, et al. (2002) ePTFEcovered stent-grafts for revision of obstructed transjugular intrahepatic portosystemic shunt. Cardiovasc Intervent Radiol 25:365-372

13. Walser EM, DeLa Pena R, Villanueva-Meyer J, et al. (2000) Hepatic perfusion before and after the transjugular intrahepatic portosystemic shunt procedure: impact on survival. J Vasc Intervent Radiol 11:913918

14. Keussen I, Ho-Young Song, Bajc M, et al. (2002) Changes in the distribution of hepatic arterial blood flow following TIPS with uncovered stent and stent-graft: an experimental study. Cardiovasc Intervent Radiol 25:314-317

15. Bureau C, Otal P, Chabbert V, et al. (2002) Segmental liver ischemia after TIPS procedure using a new PTFE-covered stent. Hepatology 36: 1554

16. Brountzos EN, Alexopoulou E, Koskinas I, et al. (2000) Intraperitoneal portal vein bleeding during transjugular intrahepatic portosystemic shunt: treatment with stent-graft placement. Am J Roentgenol 174:132134

17. Rose JDG, Pimpalwar S, Jackson RW (2001) A new stent-graft for transjugular intrahepatic portosystemic shunts. Br J Radiol 74:908912

18. Maleux G, Pirenne J, Vaninbroukx, et al. (2004) Are TIPS stent-grafts a contraindication for future liver transplantation? Cardiovasc Intervent Radio 7:140-142 